\title{
Realistic Mobility and Propagation Framework for MANET Simulations
}

\author{
Mesut Güneş, Martin Wenig, and Alexander Zimmermann \\ Chair of Computer Science, Informatik 4, RWTH Aachen University \\ \{guenes, wenig, zimmermann\}@i4.informatik.rwth-aachen. de
}

\begin{abstract}
Two main steps on the way to more realistic simulations of mobile ad-hoc networks are the introduction of realistic mobility and sophisticated radio wave propagation models. Both have strong impact on the performance of mobile ad-hoc networks, e.g. the performance of routing protocols changes with these models.

In this paper we introduce a framework which combines realistic mobility and radio wave propagation models. Our approach consists of a zone-based mobility generator and a high accuracy radio wave propagation model.

For the mobility generation a wide variety of well understood random mobility models is combined with a graph based zone model, where each zone has its own mobility model. To achieve a realistic radio wave propagation model a ray tracing approach is used. The integration of these two techniques allows to create simulation setups that closely model reality.
\end{abstract}

\section{Introduction}

A mobile ad-hoc network is created by a collection of nodes which communicate using radio interfaces and do not rely on any pre-installed infrastructure. Furthermore, it is supposed that ad-hoc networks are inherently adaptive and auto-configured. Therefore, ad-hoc networks offer immense flexibility.

In recent years the interest in the deployment of ad-hoc networks for real world scenarios grew. Still the number of real world ad-hoc networks is quite low and most of the testbeds [1 consist only of a small number of nodes. The development and testing of new algorithms and methods nowadays relies heavily on network simulations. Simulating wireless networks, and especially mobile adhoc networks, is not a trivial task and consequently there have been discussions about the validity of presented simulation results 2]3. This work does not deal with the methodological background used to analyze the output of the simulation, instead it deals with the simulator's accuracy. A key factor of accurate simulation results are accurate simulation models. To the belief of the authors the main weak points are 1) the unrealistic assumptions concerning the radio wave propagation [2, 2) the currently used simplistic mobility models 45] and 3 ) the assumed workload of the network. This work proposes a solution to the first two mentioned problems. 
Our contribution in this work is an integrated framework which allows the definition and control of the movement and the radio wave propagation model in higher detail than previous approaches. We propose a generation process which based on partitioning the simulation area into zones with different independent mobility models together with a high accuracy radio wave propagation model.

The need for such a generation framework might be illustrated by a quick literature overview: Taking the publications of the MobiHoc conferences of the last two years as an example, it is obvious that there is a need for better tool support for simulation designers. Out of 52 papers 35 presented simulation results (around 67\%). Six papers did not give any information about the used mobility model, 10 used random waypoint to model mobility and 14 considered static scenarios. Only two papers showed results obtained from considering more than one mobility model. Only two papers mention the used radio wave propagation model, ten papers gave no indication about the used model and 22 used a fixed radius. Assuming that all papers which did not specify their propagation model used a fixed range it can be concluded that all papers used circular, bidirectional links. None of the presented papers used a small scale (fading) model.

The structure of the paper is as follows: In section 2 mobility and radio wave propagation models are presented. In section 3 our approach is discussed in detail and in section 4 some simulation results obtained with ns-2 are discussed.

\section{Related Work}

\subsection{Random Mobility Models}

There are many random mobility models proposed in literature. Detailed descriptions of these models are given in 6778910. The most simple random mobility model is called Random Walk. In this model, a node selects randomly a direction and speed from predefined ranges $\left[\varphi_{\min }: \varphi_{\max }\right]$ and $\left[v_{\min }: v_{\max }\right]$, respectively. Each movement is bounded either by travel time or distance.

The Random Waypoint mobility model is an extension of Random Walk and integrates a pause time between two consecutive moves. A disadvantage of this model is the concentration of nodes in the center of the simulation area [1]. Besides these entity mobility models, there are group mobility models which specify how a set of nodes move in respect to each other [6]. In the Nomadic Community Mobility Model, all mobile nodes move to the same location in the same order but by using different entity mobility models. The Reference Point Group Mobility model specifies the movement of the group as well as the movements of the nodes within the group.

There are also models which match the characteristics of car movements. In the Freeway model, there is at least one lane in each direction of a street. The nodes move on the lanes. The speed of a node depends on other nodes on the same lane. In the Manhattan model the lanes are organized around blocks of buildings. A node can change its direction only at intersections.

All mobility models discussed so far share the assumption that there are no obstacles. In 12 a refinement of random mobility models by integrating obstacles 
is proposed. The obstacles represent buildings. Upon the definition of buildings, paths between them are calculated. The mobile nodes are randomly distributed on the paths and the destinations of the nodes are selected randomly among the buildings. The nodes move on the defined paths from building to building. Additionally, the communication characteristic is also affected by the obstacles. A mobile node inside a building cannot communicate with a mobile node outside the building.

\subsection{Models from Cellular Network Research}

In cellular networks the geographical area is divided into cells. There is a base station in each cell which provides communication service for the nodes. The mobility models in this area describe the mobility of the nodes regarding the cell topology, i.e., when a node moves from a particular cell to another cell. In 13 a hierarchy of such models with regard to metropolitan, national, and international mobility is presented. Their results cannot directly be applied to MANET simulations, since here movements have to be described with higher granularity.

\subsection{Mobility Models from Real User Traces}

The best input for simulations would be derived from real traces. However, it is very difficult for the research community to obtain those data. Therefore, there are few studies reported which are based on real data [14. In [15] the authors describe how real user traces can be used to build simulation models. It is based on the trace collection at Darthmouth College. Their interesting research can be used as input for our mobility generator. But using it to evaluate our models is not meaningful, since we could set up our model to deliver similar results by simply creating a similar geometry and using their parameters as input.

\subsection{Radio Wave Propagation}

Radio channels are more complicated to model than wired channels. Their characteristics may change rapidly and randomly and they are dependent on their surrounding (buildings, terrain etc.). Nevertheless, most wireless network simulators use very simplified propagation models. In general, propagation models can be characterized into two groups: large-scale and small-scale propagation models. Large-scale models characterize how the transmission power between two nodes changes over long distances and over a long time. Small-scale models account for the fact that small movements (in the order of the wavelength) may have large influence on the transmission quality. Also, due to multipath propagation, the signal varies heavily even if the nodes do not move.

Common used propagation models are the Free Space model, the Two-Ray Ground model and the Shadowing model [16. In addition, Ricean and Rayleigh fading are often used as small-scale models [17. None of these models is able to correctly model complex scenarios with obstacles. One way to overcome this 
limitation is the use of ray tracing technologies. In [18] an approach using this technique is described. It allows the definition of obstacles in a graphical editor and this scenario description is used in the simulation to feed a ray tracing algorithm. The algorithm is started once for every new position the node takes up. The authors state that this approach slows down the simulation by a factor of up to 100. Also, no movement information is generated by this tool.

Other approaches 1920 either do not scale well or their accuracy is highly dependent on the selected grid resolution of the calculated scenario. Additionally, these models have been developed for fixed wireless networks.

\section{Generation Framework}

The proposed framework named CosMos addresses the generation of i) realistic mobility patterns, and ii) accurate radio wave propagation information for the specific scenario. The whole process needs three steps. First the designer has to create a scenario for the desired simulation setup. In the next two steps the energy density maps for the radio wave propagation model are precomputed and the movement files are generated.

\subsection{Scenario Creation}

The scenario consists of movement zones (MZ) and obstacle zones (OZ). Both can basically be described as polygons and they divide the simulation area into smaller parts. The designer assigns a mobility model to each MZ. All models have their own set of parameter (e.g. maximum speed) and are independent of each other. When MZs overlap nodes can change from one zone to the other. The probability to leave the current zone can also be set by the designer.

The obstacles have three parameters: their height, their reflection, and transmission coefficients. The position of the zones can be set up by the designer as wished, e.g to model an indoor scenario. The values for the mobility models must be decided individually according to the intended scenario. Here, only very limited experience has been gathered by researchers. The approach presented in [15] can be integrated in our framework. For the radio wave propagation model there have been measurements which can be used, e.g. 16. Another approach is to perform own measurements and use these as input for the ray tracer.

\subsection{Mobility Generator}

The movement zones create a weighted and directed graph: the zones are the vertices $(\mathcal{V})$, there is an edge between two vertices if the corresponding movement zones overlap. The set of edges $(\mathcal{E})$ has weights attached to them. The function $w: \mathcal{E} \rightarrow(0 \ldots 1)$ defines weights for all edges of the graph $G(\mathcal{V}, \mathcal{E})$. The weight $w_{i, j}$ of a directed edge $e_{i, j} \in E$ from zone $i$ to zone $j$ corresponds to the probability for nodes to leave zone $i$ to zone $j$. 
Initially all nodes are distributed randomly in the movement zones. During the generation process nodes move inside the zones according to the mobility model of their current zone. If a node decides to leave to a neighbouring zone (depending on the exit probability) it moves towards the overlapping region of the two zones. When it arrives there, the mobility model of the new zones takes over and the node starts to move according to the new mobility model.

Our approach allows the calculation of the spatial distribution of nodes on the simulation area as well as the distribution of nodes on the movement zones. Since initially all nodes are distributed randomly in all movement zones, there will be a point in time in which zones with a high exit probability will 'loose' some nodes to the zones with a lower exit probability. After a while, the distribution of nodes should become stable. Figure 1 shows a small example with three movement zones: two large rectangles on each side and a narrow street connecting them. Zone A has a higher exit probability than Zone B. The street is only used to travel from zone A to B and vice versa.

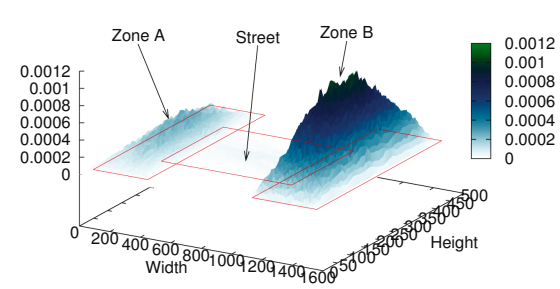

(a) Spatial distribution of the nodes.

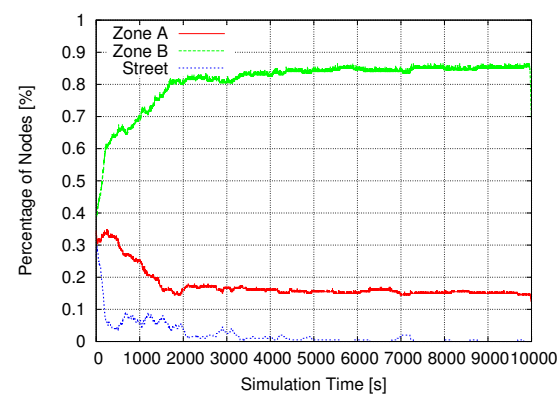

(b) Distribution of the nodes on the zones.

Fig. 1. Visualizing the steady state

Figure 1(a) shows the (long-term) spatial probabilities of the simple scenario. It shows the probability of a node being at a specific place over a simulation run of 10000 seconds. Figure 1(b) shows that initially all zones contain approximately one third of all nodes. During the simulation run the distribution slowly changes: all nodes in the street zone leave this zone to one of the neighbouring zones. The distribution stabilizes when most of the nodes are inside the zone with the lower exit probability. Nevertheless, still nodes travel from one zone to the other, but the long term average remains relatively constant from second 2000 onwards. If this 'steady-state' behaviour is important for the considered simulation scenario then the first seconds will have to be cut off.

The proposed framework per default creates several independent variants of the movements according to the given models. This helps the researcher to conduct simulations with independent replications. 


\subsection{Radio Wave Propagation Model}

The radio wave propagation model used in this work is based on a ray tracing approach. The obstacles defined in CosMos are used as input for the ray tracer. Triggering a ray tracing run for every position of the current sender is unfeasible in mobile ad-hoc networks. Instead, our approach uses a set of predefined starting points for the ray tracing approach. The ray tracer is then started once for each of this points creating an energy distribution map for each one. During the simulation the energy distribution between the sender and the receivers is calculated using weighted interpolation, as detailed below. The ray tracer accounts for the following propagation phenomenas: reflection, diffraction, and scattering.

To use the generated energy distribution maps during the simulation, we modified the ns-2 network simulator 21. We added a propagation model which reads in a given set of maps and the corresponding starting points. During the simulation, whenever a node $n_{t}$ wants to transmit a packet, a $k$-nearest neighbor search is started 1 . This search finds the $k$ nearest starting points and their corresponding energy distribution maps to the sender's position. For each node inside the maximum interference range of an unobstructed radio wave the transmission power is calculated. The formula used for the weighted interpolation is given below:

$$
s_{t-r}=\frac{\sum_{i=0}^{k-1} \frac{s_{i}}{\left\|p o s_{i}-\operatorname{pos}_{t}\right\|^{p}}}{\sum_{i=0}^{k-1} \frac{1}{\| \operatorname{pos}_{i}-\text { pos }_{t} \|^{p}}},
$$

where $s_{t-r}$ is the signal strength between the transmitter node $n_{t}$ and the receiver node $n_{r}$. The position of the transmitter is given as pos ${ }_{t}, \operatorname{pos}_{i}$ denotes the position of the starting point of the $i$-th closest map. Note that $s_{i}$ is the predicted signal strength of map $i$ at the position of the receiver $\operatorname{pos}_{r}$. The exponent $p$ controls how much influence is given to further away map: 2 .

The benefits of our approach are that it is not necessary to rerun the ray tracing algorithm during simulation time, it is not necessary to divide the simulation area into evenly sized squares, and the accuracy can be increased in areas with a lot of obstacles, simply by adding more starting points. A real-time evaluation tool has been developed to show the result of the interpolation. Our approach increases the simulation speed and allows the designer to choose between high accuracy and reduced memory needs 22 .

\section{Results}

In this Section we discuss some simulation results created with ns-2. The simulation scenario was created with CosMos. The presented Scenario models the office building in which the authors' chair is in. The intention of the studies was

\footnotetext{
${ }^{1}$ Our experiments showed $k$ equal to 3 gave good results.

${ }^{2}$ In our experiments $p$ was set to 3 .
} 


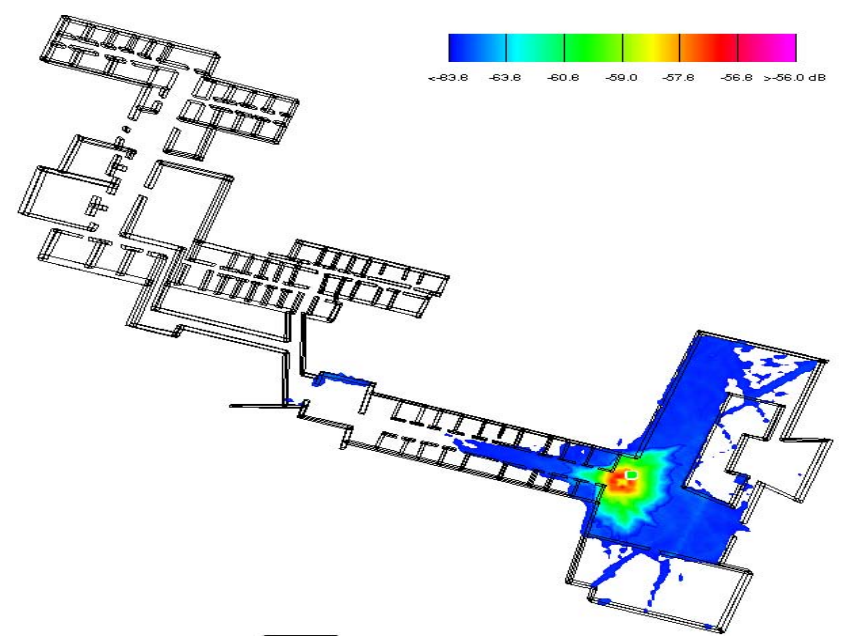

Fig. 2. Indoor scenario

to show the impact of the mobility and radio wave propagation models on the performance of MANET routing protocols.

Figure 2 shows the scenario outline. Only the ground floor is modeled here. All nodes are equipped with IEEE 802.11 radio interfaces with a transmission rate of $11 \mathrm{Mbit} / \mathrm{s}$ and a transmission power of $0.1 \mathrm{~mW}$. The receiving threshold was set to $-88 \mathrm{dBm}$, a value taken from the specification of the Cisco Aironet $1240 \mathrm{AG}$ Series access point. The AODV implementation of the university of Uppsala [23] and the current version of DSR [11] in ns2 were used. Thirty connections between randomly selected nodes were started, each one offering $32 \mathrm{kBytes}$ of load. Movements inside of offices are seldom and relatively slow (max. speed was set to $1 \mathrm{~m} / \mathrm{s}$ ). Movements inside of the hallways on the other hand are faster and follow the freeway model (max. speed was set to $2 \mathrm{~m} / \mathrm{s}$ ). Since we had detailed plans of our building, we were able to model it with high detail. To check the accuracy of the radio wave propagation model, we conducted some measurements with real-life systems and compared the results to the calculated values. The mean error between the predicted values and the calculated is $3.5 \mathrm{~dB}$ which is as good as the results for approches mentioned in section 2 .

We conducted simulations using the AODV and the DSR routing protocols in which the following combinations were considered: CosMos mobility model together with Two-Ray Ground propagation model and CosMos mobility model with ray tracing propagation model. Figure 3 shows a comparison of the throughput achieved using AODV and DSR in the presented scenario. It is clear to see that the measured values without the ray tracer propagation model can be considered as equal. But using the ray traced propagation model, the DSR protocol suffers more heavily from performance loss. AODV seems to be able to cope better with the situation. The decreasing performance for larger number of nodes can be explained by the higher number of hops between sender and destination. 
The routes are getting longer because the node density is higher and farther away nodes can also be reached. The reason for the worse performance of DSR compared to AODV seems to be a larger number of discovered paths which were actually already invalid (stale paths) when they should be used for the first time.

Figure 4 compares the average end-to-end delay for packets between source and destination. As expected, the values using TwoRay Ground can again be considered as equal. Using the ray tracer the delay of course grows due to longer routes, higher number of transmission errors, and thus higher routing overhead. Again, we see a strong influence on DSR. As a rule of thumb, one can say that if more than $90 \%$ of all packets have a delay of less than $150 \mathrm{~ms}$, VoIP is possible with reasonable quality. If scenarios with more than 60 nodes are considered, DSR is not able to fullfil this criterion. This is yet another example why accurate simulation models are absolutely neccesarry. If one would have based the decission on the simple simulation setup both algorithms would have been judged as equal but in reality only AODV is actually able to fullfil the delay bound.

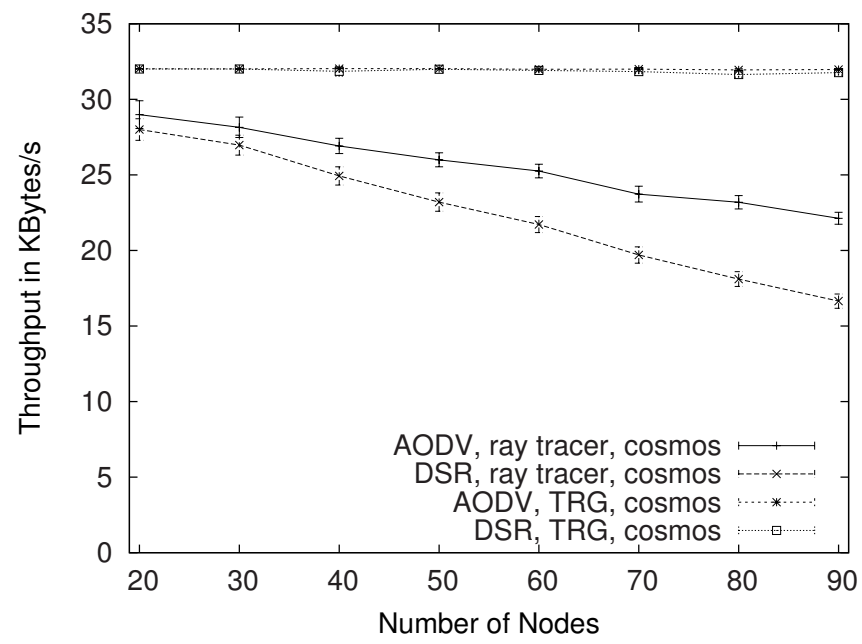

Fig. 3. Throughput comparison between AODV and DSR

Another result of our simulation study is that the mobility model is more important for larger scenarios. The smaller the simulation area compared to the transmission range of the nodes, the smaller the influence of the mobility model. We also measured the run-time of the simulations with and without our propagation model. Table 1 shows the times for the indoor simulation. The increase in run-time is relatively small since during the simulation runtime only lookups in the $k d$-tree have to be done. The preprocessing time, namely the time needed to create the energy distribution maps, is dependent on the complexity of the scenario. For the presented indoor scenario 112 starting points were used and the ray tracer needs around 12 seconds for each point (shooting 50000 photons). 


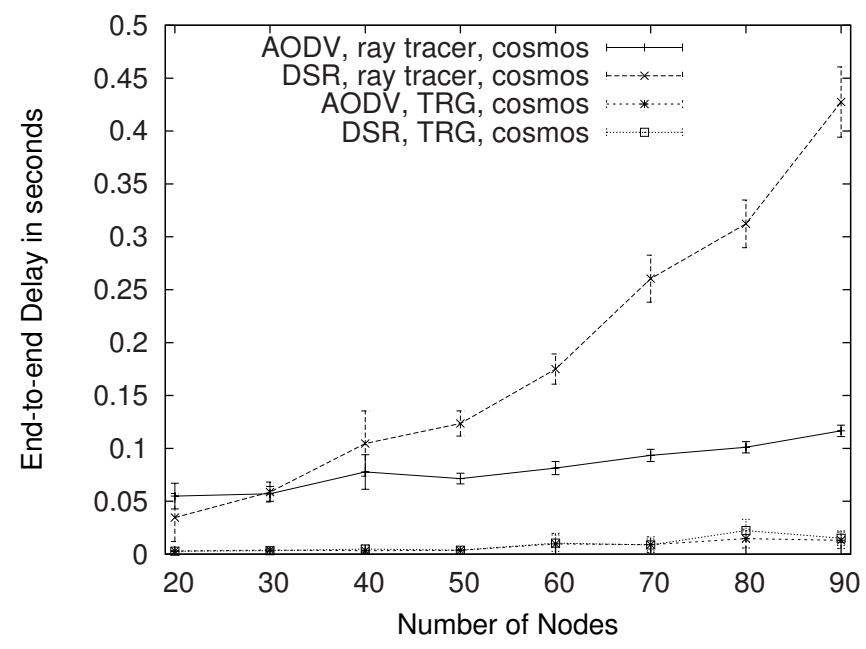

Fig. 4. Delay comparison between AODV and DSR

Table 1. Runtime of the ns-2 simulator

\begin{tabular}{|l|l|l|l|}
\hline $\begin{array}{l}\# \\
\text { Nodes }\end{array}$ & $\begin{array}{l}\text { Runtime (s) } \\
\text { TwoRayGround }\end{array}$ & $\begin{array}{l}\text { Runtime }(\mathrm{s}) \\
\text { CosMos }\end{array}$ & Factor \\
\hline 10 & 13.6 & 16.5 & 1.2 \\
\hline 20 & 34.3 & 61.9 & 1.8 \\
\hline 30 & 59.3 & 91.1 & 1.5 \\
\hline 40 & 69.1 & 119.2 & 1.7 \\
\hline 50 & 90.2 & 147.5 & 1.6 \\
\hline
\end{tabular}

\section{Conclusion}

In this paper we have introduced a mobility and radio wave propagation scenario generator for MANET. The goal was to aid researchers in the design of realistic simulation scenarios. The framework is very general and can be deployed to design scenarios with special requirements. Our approach combines a wide variety of well understood random mobility models with a graph based zone model and a sophisticated ray traced radio wave propagation model. Each zone can have a different mobility model. The framework allows to generate the mobility definition and the ray tracer results from one common scenario. So the combination of realistic movement models and accurate radio wave propagation models becomes an easy task for the researcher. Furthermore, our approach allows the calculation of the spatial distribution of nodes on the simulation area as well as the distribution of the nodes on the defined zones. This allows us to figure out the time when the stationary state is reached. Since, trustworthy MANET simulations should begin when the stationary state is reached. 


\section{References}

1. Güneş, M., Bouazizi, I.: From Biology to Technology: Demonstration Environment for the Ant Routing Algorithm for Mobile Ad-hoc Networks. In: Tenth Annual Int. Conference on Mobile Computing and Networking (ACM MobiCom 2004), Philadelphia, USA (September 2004)

2. Kotz, D., Newport, C., Gray, R.S., Liu, J., Yuan, Y., Elliott, C.: Experimental evaluation of wireless simulation assumptions. Technical Report TR2004-507, Dept. of Computer Science, Dartmouth College (June 2004)

3. Pawlikowski, K., Jeong, H.D.J., Lee, J.S.R.: On credibility of simulation studies of telecommunication networks. IEEE Communications 40(1) (January 2002) 132139

4. Bettstetter, C., Hartenstein, H., Perez-Costa, X.: Stochastic properties of the random waypoint mobility model: epoch length, direction distribution, and cell change rate. In: MSWiM '02: Proc. of the 5th ACM int. Workshop on Modeling, Analysis and Simulation of Wireless and Mobile Systems, NY, USA, ACM Press (2002) $7-14$

5. Bettstetter, C., Resta, G., Santi, P.: The node distribution of the random waypoint mobility model for wireless ad hoc networks. IEEE Trans. Mobile Computing 2(3) (2003) 257-269

6. Hong, X., Gerla, M., Pei, G., Chiang, C.C.: A group mobility model for ad hoc wireless networks. In: Proc. of the ACM Int. Workshop on Modeling and Simulation of Wireless and Mobile Systems (MSWiM). (August 1999) 53-60

7. Camp, T., Boleng, J., Davies, V.: A survey of mobility models for ad hoc network research. Wireless Communications and Mobile Computing (WCMC): Special issue on Mobile Ad Hoc Networking: Research, Trends and Applications 2(5) (2002) 483-502

8. Sanchez, M.: Mobility models. http://www.disca.upv.es/misan/mobmodel.htm (2005)

9. Bai, F., Sadagopan, N., Helmy, A.: The important framework for analyzing the impact of mobility on performance of routing for ad hoc networks. AdHoc Networks Journal - Elsevier Science 1(4) (November 2003) 383-403

10. Lin, G., Noubir, G., Rajaraman, R.: Mobility models for ad hoc network simulation. In: Proc. of the 23rd Conference of the IEEE Communications Society, Hon Kong, IEEE, IEEE (March 7-11 2004)

11. Johnson, D.B., Maltz, D.A.: Dynamic source routing in ad hoc wireless networks. In: Mobile Computing. Volume 353. Kluwer (1996)

12. Jardosh, A., Belding-Royer, E.M., Almeroth, K.C., Suri, S.: Towards realistic mobility models for mobile ad hoc networks. In: The Ninth Annual Int. Conference on Mobile Computing and Networking (ACM MobiCom 2003), San Diego, USA, ACM (September 14-19 2003)

13. Lam, D., Cox, D.C., Widom, J.: Teletraffic modeling for personal communications services. IEEE Communications Magazine 35 (Feb. 1997) 79-87

14. Hsu, J., Bhatia, S., Takai, M., Bagrodia, R., Acriche, M.J.: Performance of mobile ad hoc networking routing protocols in realistic scenarios. In: MilCom 2003, Boston, Massachusetts (October 13-16 2003)

15. Kim, M., Kotz, D., Kim, S.: Extracting a mobility model from real user traces. In: Proc. of the 25th Joint Conference of the IEEE Computer and Communications Societies, Barcelona, Spain, IEEE Computer Society Press (April 2006) 
16. Rappaport, T.S.: Wireless Communications, Priciples \& Practice. Prentice Hall (1999)

17. Punnoose, R.J., Nikitin, P.V., Stancil, D.D.: Efficient simulation of ricean fading within a packet simulator. In: Vehicular Technology Conference. (Sep. 2000)

18. Dricot, J.M., Doncker, P.D.: High-accuracy physical layer model for wireless network simulations in ns-2. In: Proc. of the Int. Workshop on Wireless Ad-hoc Networks. (2004)

19. Catedra, M., Perez, J., de Adana, F.S., Gutierrez, O.: Efficient ray-tracing techniques for three-dimensional analyses of propagation in mobile communications: application to picocell and microcell scenarios. Antennas and Propagation Magazine 40(2) (Apr 1998) 15-28

20. Schmeink, M., Mathar, R.: Preprocessed indirect 3D-ray launching for urban microcell field strength prediction. In: AP 2000 Millennium Conference on Antennas and Propagation. (April 2000)

21. Fall, K., Varadhan, K.: The ns-2 manual. Technical report, The VINT Project, UC Berkeley, LBL and Xerox PARC (2003)

22. Schmitz, A., Wenig, M.: The effect of the radio wave propagation model in mobile ad hoc networks. In: MSWiM '06: Proc. of the 9th ACM international workshop on Modeling, Analysis and Simulation of wireless and mobile systems, Torremolinos, Spain, ACM Press (2006)

23. Wiberg, B., Nordström, E.: Ad-hoc on-demand distance vector routing - for real world and simulation. http://core.it.uu.se/core/index.php/AODV-UU (October 2006) 